\title{
On-Air Character Recognition System For Visually Impaired People
}

\author{
${ }^{1} \mathrm{~K}$. Preethi, ${ }^{2} \mathrm{~S}$. Chithra \\ ${ }^{1,2}$ Department of Information Technology, \\ Sri Sivasubramaniya Nadar College of Engineering, Kalavakkam, Chennai-603110, \\ preethimpk31@gmail.com,chitras@ssn.edu.in
}

\begin{abstract}
Character recognition is a process which allows computers to recognize written or printed characters such as numbers or letters and to change them into a form that the computer can use. The character recognition system used to develop cost effective assistive technologies to provide blind people with greater degree of confidence in their activities. OnAir communication is developed by using the sensors which helps the blind people to recognize the character easily. The purpose of this paper is to take handwritten characters as input, process the character, train and test the character using machine learning method to recognize the pattern and character. Thus, it has been planned to design a device using sensors to recognize the characters written on air. The device is equipped with gyroscope and accelerometer to recognize the hand movement. While there is some on-air writing recognition system which uses wireless sensor- equipped pens. However, this project is planned for the use of visually impaired people to recognize the character.
\end{abstract}

Index Terms-Accelerometer, Gyroscope, Arduino, Matlab, Connecting Wires.

\section{INTRODUCTION}

In the recent years Handwritten character recognition system has been one of the fascinating and challenging research areas in field of technology. These challenges open a new direction for human computer interaction. It is a way for computers to understand human body language. It can improve the interface between human and machine is crucial to facilitating this interaction in numerous applications. Several research works have been focusing on new techniques and methods. It would reduce the processing time while providing higher recognition accuracy. This system make the blind people to write the character on air using sensors to improve accuracy of recognition. Traditional character recognition system is widely applied to such problems as converting scanned books to text and converting images. In traditional system the character recognition is based on the use of images which require more memory and effort for recognition.

On-Air communication is planned to be developed by using the sensors which helps the blind people to recognize the character easily. A way for computers to understand human body language, thus building a richer bridge between machines and humans than primitive text user interface. The accelerometer and gyroscopic sensors are capable of capturing 3D finger positions and movements. This is the way to control and interact with computers by moving fingers in the air. The positions of fingers are precisely captured by a computer device by tracking the moving patterns of fingers to recognize the character.

MPU6050 sensor module is complete 6-axis Motion Tracking Device which combines 3-axis Gyroscope and 3-axis Accelerometer to tracking the movement of the object. It consist of $\mathrm{I} 2 \mathrm{C}$ bus interface to communicate with the microcontrollers. It has Auxiliary I2C bus to communicate with other sensor like 3-axis Magnetometer, Pressure sensor etc. If 3-axis Magnetometer is linked to I2C bus, then MPU6050 can provide complete 9-axis Motion Fusion output.

\section{A. Acronyms 3-Axis Gyroscope}

The MPU6050 has 3-axis Gyroscope with Micro Electro Mechanical System technology and it is used to detect rotational velocity for $\mathrm{X}, \mathrm{Y}$ and $\mathrm{Z}$ axes. When the gyroscope are rotated about any of the sense axes then the Effect causes a vibration is detected by a MEM inside MPU6050. The resulting signal is amplified, demodulated, and filtered to produce a voltage is directly proportional to the angular rate. This voltage is digitized by using 16-bit ADC to sample each axis. The full-scale range of output to be like $+/-250,+/-500$, +/- 1000, +/- 2000 .

\section{A. 3-Axis Accelerometer}

The MPU6050 consist of 3-axis x,y and $z$ with Micro Electro Mechanical technology and it is used to detect the angle of inclination for the axis $\mathrm{X}, \mathrm{Y}$ and $\mathrm{Z}$. Acceleration along the $\mathrm{X}, \mathrm{Y}$ and $\mathrm{Z}$ axes deflects the movable mass. This displacement of moving plate unbalances the differential capacitor which results for the sensor output. This output amplitude is proportional to acceleration. The 16-bit analog to digital convertor is used to get digitized output. The full-scale range of acceleration are $+/-2 \mathrm{~g},+/-4 \mathrm{~g},+/-8 \mathrm{~g},+/-16 \mathrm{~g}$. It measured as gravity force unit. When device placed on flat surface it will measure $0 \mathrm{~g}$ on $\mathrm{X}$ and $\mathrm{Y}$ axis and $+1 \mathrm{~g}$ on $\mathrm{Z}$ axis.

The rest of the paper is structured as follows. In Section 2, the various survey works can be provided. In Section 3, system architecture and algorithm for proposed system. In Section 4, implementation, necessary requirements and results . In section 5, the paper is concluded with the future work. 


\section{RELATED WORK}

In the literature survey, it has been identified that various challenging issues on character recognition for visually impaired people. In this section we review recent research on various applications and sensors.

\section{A. Motion Sensors Application Gestures in the Air Recognizing}

Here, Maria Atiq Mirza , Nayab Gul Warraich has discussed about the methods involved for fine tuning of this sensor and further using this sensor to convert the handwriting to an editable document. Converting ideas from scribbled down notes to a useful soft copy is a time consuming task. A pen that employs its motion to transfer the writing into an editable word document is a key to this problem. It proposes the use of the dynamics of writing process as a solution to the problem. The device has accelerometer and gyroscope sensors fused together for recording the motion of the pen. The raw data from the motion sensors is converted to a processed useful data by implementing a number of error corrections to reconstruct the word.

\section{B. Gestures In The Air Recognizing}

Sharad Vikram, Lei Li, Stuart Russell in this paper says that the recent technologies in vision sensors are capable of capturing 3D finger positions and movements. It proposes a novel way to control and interact with computers by moving fingers in the air. The positions of fingers are precisely captured by a computer vision device. Through tracking the moving patterns of fingers, we can recognize users' intended input information. It demonstrate this human input approach through an example application of handwriting recognition. By treating the input as a time series, it propose a fast algorithm using dynamic time warping to recognize characters in online fashion.

One technique used in the process is the segmentation of the data points. This is difficult as it is hard to determine the beginning and end of segments, so typically unsupervised learning and data-driven approaches are used. The statistical approaches to this problem use Hidden Markov Models or use a combination of HMMs and neural networks to recognize characters. Hilbert Warping has been proposed as an alignment method for handwriting recognition. Other scenarios have been

proposed, including one where an LED pen is tracked in the air. This allows for 3D data to be interpreted, but also makes sure that the beginning and end of input are clearly defined. Finally, treating the handwriting problem like speech recognition, i.e. treating the input points as a signal but the same problem of segmentation arises. These techniques have problems with accuracy in identification[2].
C. Modeling and Recognition of Characters, Words, and Connecting Motions

Mingyu Chen, Ghassan AlRegib and Biing-Hwang Juang in this paper says that the Air-writing is fundamentally different from conventional handwriting on paper or a surface, which provides no haptic feedback. Similar to motion gestures, air-writing is tracked with a continuous stream of sensor data, and the writing is intuitively rendered in the air in unistroke without any pen-up and pen-down information. The user envisions a writing box in the space and writes in this imaginary space without haptic feedback. Air-writing also does not require visual feedback. Air writing consists of two levels: motion characters and motion words. Motion characters are isolated alphanumeric letters written in one continuous stroke.

Hidden Markov models (HMMs) are widely used for online handwriting recognition. In ligature models are proposed to address online recognition of cursive handwriting, in which successive letters are connected without explicit penup moves. Motion based handwriting can be consider as parallel to motion gestures or sign. Many sign language recognition systems use HMMs with various sensing technologies, such as data gloves and vision-based techniques. In air-writing recognition was achieved with inertial sensors attached to a glove. In finger writing in the air is tracked with a depth sensor. Different motion sensing and tracking technologies impose various behavioral load on the user. As an example, wearing data gloves is often considered by many users as an undesirable burden and may change the wearing user's motion behavior[3].

\section{Leap Motion Controller}

consecutively with equation numbers in parentheses flush with the right margin, as in (1). First use the equation editor to create the equation. Then select the "Equation" markup style. Press the tab key and write the equation number in parentheses. To make your equations more compact, you may use the solidus ( $/$ ), the exp function, or appropriate exponents. Use parentheses to avoid ambiguities in denominators. Punctuate equations when they are part of a sentence, as in

\section{PRoposed System}

The architecture figurel displays the consecutive steps involved in the processing of on-air character recognition. This given schematic representation proves to serve as an efficient guide for blind people.

To recognize the character on air, the sensor is fixed over the finger. After the setup the character is drawn on the air based on the finger movement. The sensor reads the character and the values are measured as yaw, pitch and roll. The values are trained and tested using machine learning and the character is recognized. 


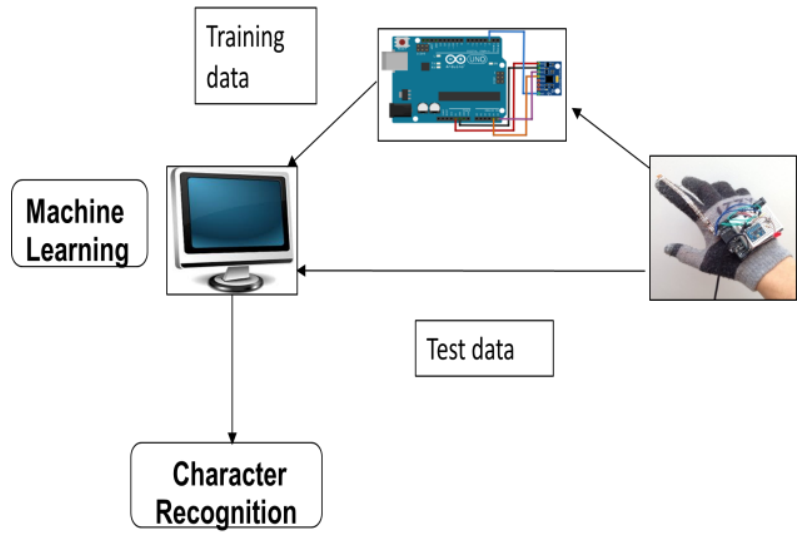

Fig.1. Proposed Architecture For Character Recognition.

\section{A. Interfacing the Arduino and MPU6050}

The MPU6050 communicates with the Arduino through the I2C protocol in which the MPU6050 is connected to Arduino. If MPU6050 module has a $5 \mathrm{~V}$ pin, then it can connect to Arduino's $5 \mathrm{~V}$ pin. If not, it has to connect to the $3.3 \mathrm{~V}$ pin. And the GND pin of the Arduino is connected to the GND of the MPU6050.

MPU-6050 sensor combines a gyroscope and accelerometer. An accelerometer works on the principle of the piezoelectric effect. Gyroscopes are used to measure angular rate quickly on an object turns. The rotation is typically measured in reference to one of three axes: yaw, pitch and roll.

- Yaw : It measures rotation about other axes by appropriate mounting orientation.

- Pitch : It provides position in degrees.

- Roll : When mounting on its side yaw axis become the roll axis.

In Arduino, the Code for MPU6050 sensor is written in order to find the yaw, pitch and roll values of the given character. The values of yaw, pitch and roll for all the character are stored in Excel file as a dataset. Then import the values which are stored in excel file and assign the variable names in MATLAB. A separate code is written for single and multiple stroke and run the program accordingly. Then the graph will be plotted and displayed as a character.

TABLE I

UNITS FOR MAGNETIC PROPERTIES

\begin{tabular}{|c|c|c|}
\hline Benefits & Existing System & Proposed System \\
\hline Expensive & High & Low \\
\hline Accuracy & Low & Moderate \\
\hline $\begin{array}{c}\text { Time } \\
\text { Consumption }\end{array}$ & High & Low \\
\hline Storage Space & High & Moderate \\
\hline
\end{tabular}

\section{RESULTS AND DISCUSSION}

The system helps to analyze the set of values by using the Arduino and the graphical representation are displayed with the help of Matlab. Among the 26 alphabetical characters, the
15 characters were drawn successfully and the values are collected over a period of time.

The accelerometer and gyroscope sensor is connected with the Arduino UNO board by the help of the jumper wires. It is holded and kept at the palm of the human. Then, the setup is linked with the USB cable and is further attached with the Arduino IDE software.

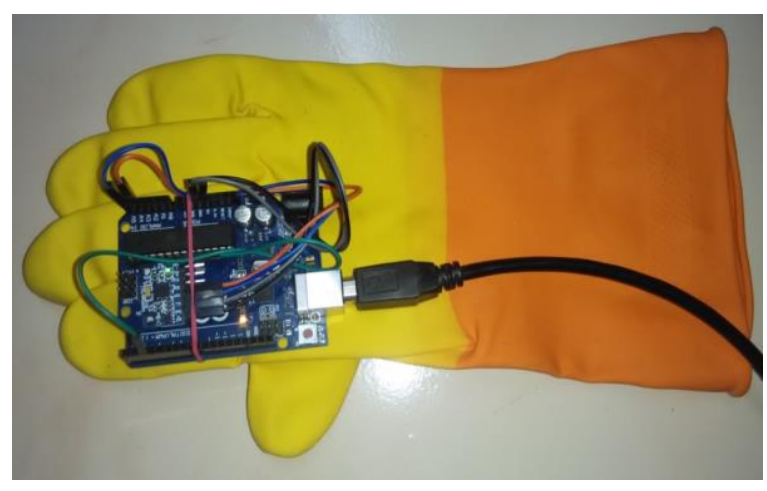

Fig.2. Arduino Setup

The sensor is moved on the air for writing a character. For instance, if the character ' $c$ ' is written on-air. The setup will be ended up with three values namely, yaw, pitch and roll. Those values are displayed below.

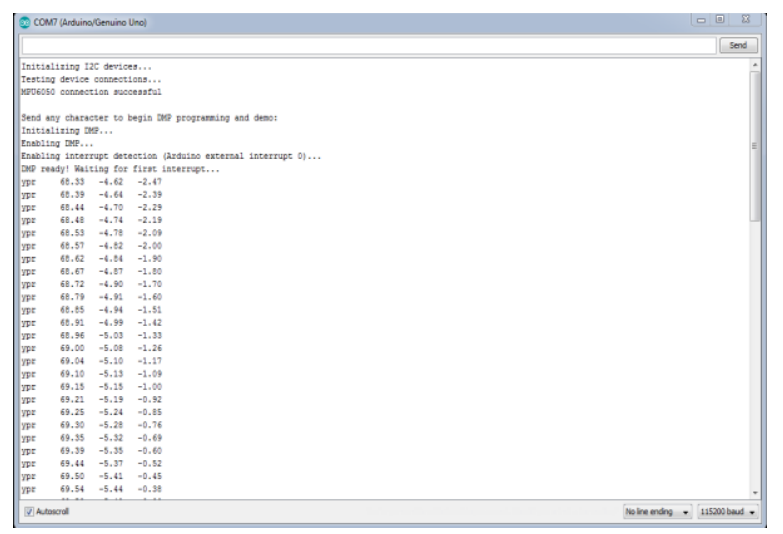

Fig.3. 'c' value display

The values of the yaw, pitch is taken and stored in excel sheet as database. And those are imported in the Matlab for plotting the values and to obtain a character.

A. Create 2-D Line Graph

Step 1: In the Command Window, define $\mathrm{x}$ as a vector of 100 linearly spaced values between 0 and $2 \pi$. Define y as the sine function evaluated at the values in $\mathrm{x}$.

Step 2: In the Workspace panel in the MATLAB desktop, select the variables to plot.

Step 3: Select the 2-D line plot from the gallery on the Plots tab.

\section{For One Stroke}

$\mathrm{x}=0: \mathrm{pi} / 100: 2 * \mathrm{pi}$

$y=\sin (x)$;

figure

plot(yy,xx) 


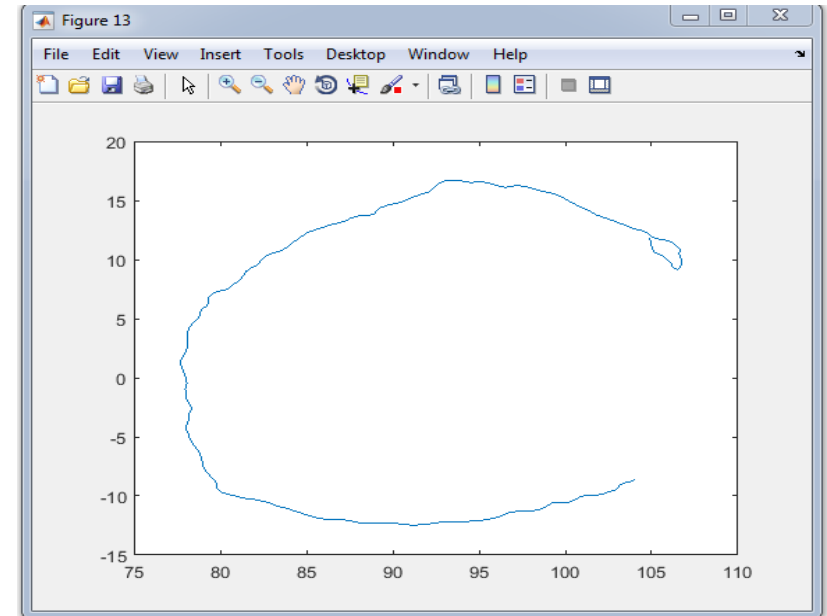

Fig. 4. Character ' $\mathrm{C}$ '

Similarly, the remaining characters are recorded and is displayed by the $2 \mathrm{D}$ line graph.

\section{V.CONCLUSION}

Thus, on-air system is able to sense both single and multistroke characters whose shape is written in air. The system has been proposed to be the preliminary phase for character recognition. In this phase, the character patterns are sensed and the points are plotted as graphical pattern to visualize the character. In the future work, machine learning techniques are used to train the datasets to improve the accuracy of recognition.

\section{REFERENCES}

[1] Maria Atiq Mirza , Nayab Gul Warraich “ Application Of Motion Sensors In Hand Writing Conversion" Conference Vol-2, No-3, July 2015.

[2] Sharad Vikram, Lei Li and Stuart Russell "Handwriting and Gestures in the Air, Recognizing on the Fly", ACM, 2013.

[3] Ning Xu, Weiqiang Wang and Xiwen Qu "Recognition Of In-Air Handwritten Chinese Character Based On Leap Motion Controller'DOI , 2015.

[4] Mingyu Chen, Ghassan Alregib and Biing-Hwang Juang "Air-Writing Recognition-Part I: Modeling and Recognition Of Characters, Words, And Connecting Motions" IEEE vol. 46, no. 3, June 2016

[5] Esra tunçer "Accelerometer based handwritten character recognition using dynamic time warping", conference 2016.

[6] Asano, T., and Honda, S, "Visual interface system by character handwriting gestures in the air". In IEEE ,2010.

[7] J.Pradeep, E.Srinivasan and S.Himavathi "Diagonal Based Feature Extraction For Handwritten Character Recognition System Using Neural Network" IEEE 2011.

[8] Shaikh Jahidabegum K"Character Recognition System for Text Entry Using Inertial Pen” IJSETR Volume 4, Issue 10, October 2015.

[9] Anuradha Salvi and Amar Deshmukh "Wireless Digital Writing Human Computer Interaction Device" conference Volume 5, Issue 4 ,July,2014.

[10] Shengli Zhou1, Zhuxin Dong,and Chung Ping Kwong"Hand-Written Character Recognition Using MEMS Motion Sensing Technology" conference July $2-5,2008$.

[11] Asano, T., and Honda, S. Visual interface system by character handwriting gestures in the air. In IEEE RO-MAN 2010.

[12] Hammond, T., and Paulson, B. Recognizing sketched multistroke primitives. ACM Oct. 2011.

[13] S. Zhang, "Handwritten Character Recognition Using Orientation Quantization Based on 3D Accelerometer"
[14] S.-D. Choi, A. S. Lee, and S.-Y. Lee, "On-Line Handwritten Character Recognition with 3D Accelerometer," IEEE conference 2006.

[15] C. Amma, D. Gehrig, and T. Schultz, "Airwriting recognition using wearable motion sensors," in Proc. 1st Augmensted Human Intl. Conf., 2010

[16] M. Chen, G. AlRegib, and B.-H. Juang, "Air-writing recognition Detection and recognition of writing activity in continuous stream of motion data," IEEE conference 2008.

[17] M. Chen, G. AlRegib, and B.-H. Juang, "Feature processing andmodeling for 6dmotion gesture recognition," IEEE Trans.Multimedia, vol. 15, no. 3, pp. 561-571, Apr. 2013.

[18] P. Isokoski and R. Raisamo, "Quikwriting as a multi-device text entry method," in Proc. 3rd Nordic Conf. Human-Computer Interaction, 2004, pp. 105-108.

[19] T. K"oltringer, P. Isokoski, and T. Grechenig, "Twostick: Writing with a game controller," in Proc. Graph. Interface, 2007, pp. 103-110.

[20] R. Plamondon and S. Srihari, "Online and off-line handwriting recognition: a comprehensive survey," IEEE Trans. Pattern Anal. Mach. Intell., vol. 22, no. 1, pp. $63-84$, Jan. 2000.

[21] J. Makhoul, T. Starner, R. Schwartz, and G. Chou, "On-line cursive handwriting recognition using hidden markov models and statistical grammars," in Proc. Workshop Human Lang. Technol., 2006, pp. 432 436. 